\title{
Concentrator location in telecommunications
}

\author{
Hande Yaman \\ Bilkent University, Department of Industrial Engineering, Bilkent 06800 Ankara, Turkey \\ (e-mail: hyaman@bilkent.edu.tr)
}

Received: October 2003

\begin{abstract}
We survey the main results in the author's PhD Thesis presented in December 2002 at the Université Libre de Bruxelles and supervised by Prof. Martine Labbé. The dissertation is written in English and is available at smg . ulb. ac . be. Several versions of concentrator location problems in telecommunication networks are studied. The thesis presents a list of polyhedral results for these problems and a branch and cut algorithm for the most general problem introduced.
\end{abstract}

Key words: Concentrator location, polyhedral theory, branch and cut

AMS classification: 90B80, 90B18, 90C57

\section{Introduction}

A generic telecommunication network consists in access networks connecting terminals (user nodes) to concentrators and a backbone network interconnecting these concentrators or connecting them to a central unit. Our thesis (Yaman 2002) is devoted to the study of concentrator location problems in telecommunication networks in which access networks are stars.

These problems arose in the context of a project funded by France Telecom. The main problem can be described as follows. Given a set of terminal nodes and a traffic matrix, the entries of which representing the amount of traffic to be routed between pairs of terminals, determine a subset of the terminal nodes that will be location of concentrators. When a concentrator is located at a terminal node, this terminal node is assigned to this concentrator. Each remaining node is assigned to exactly one concentrator node. The network connecting the concentrators is complete.

The traffic of a commodity is routed as follows: it goes from its origin to the concentrator to which the origin is assigned and then to the concentrator to which the destination is assigned and then to the destination. 
A concentrator has a fixed capacity in terms of the traffic that passes through it. This traffic is the sum of the traffic originating or destinating at nodes that are assigned to it and the traffic between these nodes and the nodes that are assigned to other concentrators.

There is a fixed cost for installing a concentrator at a certain node and a cost per unit of traffic routed between two given nodes. The aim is to locate the concentrators and to assign the remaining nodes to the concentrators to minimize the total cost of installation and routing. We call this problem Quadratic Capacitated Concentrator Location Problem with Complete Routing $(Q C L-C)$ (also known as the Capacitated Hub Location Problem with Single Assignment).

We consider two relaxations of the $Q C L-C$ based on the capacities. In the first relaxation, each concentrator has a fixed capacity in terms of the traffic adjacent at nodes assigned to it. The second relaxation is obtained by removing the capacity constraints. These two relaxations lead to linear capacitated and uncapacitated versions respectively.

We also study two special cases of the problem. The first case occurs when the routing cost on the backbone network is negligible. The second case corresponds to solving the location and routing problem on a network with a star backbone. In this case we are given a central node and each node that receives a concentrator is connected to this node by a direct link. For both special cases, we also consider the relaxations with respect to the capacity structures. This makes nine different problems which are closely related.

\section{Literature survey and relationships between the nine polyhedra}

The thesis is organized into three parts. The first part starts with the introduction and a survey on location problems which have applications in telecommunications (Gourdin et al. 2002). It includes also a chapter on formulations of the nine problems and the relationships between them. The main result is that the projections of polyhedra of problems with routing cost on the space of assignment variables correspond to polytopes of problems with no routing cost.

\section{Polyhedral analysis}

The second part which is on polyhedral study is divided into three chapters based on the capacity constraints: uncapacitated, linear capacitated and quadratic capacitated. In each chapter, we consider the location problem with no routing cost, with routing cost on a star backbone and with routing cost on a complete backbone network.

An important result is that the facet defining inequalities of the polytope of a problem with no routing cost define facets of the polyhedron of the problem with star routing or complete routing for the same capacity structure. These are in fact 
all facet defining inequalities of these polyhedra which involve only the assignment variables. We also derive some general results on facet defining inequalities which involve only the traffic variables. Then the polyhedral properties of each problem is studied separately in Labbé and Yaman (2003a, b) and Labbé et al. (2003).

\section{Computational study}

In the last part of the thesis, a branch and cut algorithm is presented to solve the $Q C L$ $C$ (Labbé et al. 2003). First, several formulations are compared from theoretical and computational points of view. Then a preprocessing algorithm is presented and is shown to be very effective to reduce the CPU time for the instances considered. A test to choose useful cuts which reduce the gap and the CPU time is also conducted. Additional tests are done to decide for the parameters of the algorithm.

Finally, computational results are presented for two sets of instances: one supplied by France Telecom and another derived from the OR Library (Beasley 1990). The results show that the algorithm can solve instances of medium size (up to 25 nodes) but it cannot prove optimality for larger problems when the capacity constraints are tight.

Acknowledgement. The research of the author was partially supported by France Telecom R\&D under contract no. 99 1B 774. Their support is gratefully acknowledged.

\section{References}

Beasley JE (1990) OR-Library: distributing test problems by electronic mail. Journal of the Operational Research Society 41: 1069-1072

Gourdin E, Labbé M, Yaman H (2002) Telecommunication and location. In: Drezner Z, Hamacher HW (eds) Facility location: Applications and theory. Springer, Berlin Heidelberg New York

Labbé M, Yaman H (2003a) Polyhedral analysis for concentrator location problems. ISRO/OR Preprint 2003/13, Université Libre de Bruxelles

Labbé M, Yaman H (2003b) Solving the uncapacitated concentrator location problem with star routing. ISRO/OR Preprint 2003/15, Université Libre de Bruxelles

Labbé M, Yaman H, Gourdin E (2003) A branch and cut algorithm for hub location problems with single assignment. ISRO/OR Preprint 2003/05, Université Libre de Bruxelles

Yaman H (2002) Concentrator location in telecommunication networks. Ph.D Thesis, Université Libre de Bruxelles. Available at http://smg.ulb.ac.be 